\title{
Onset of Intense Surface Enhanced Raman Scattering and Aggregation in the Au@Ag System
}

\author{
Priya Bhatia, ${ }^{1}$ Joseph Consiglio, ${ }^{1}$ John Diniz, ${ }^{2}$ Jia E. Lu, ${ }^{2}$ Christopher Hoff, \\ Shelby Ritz-Schubert, ${ }^{1}$ and Roger H. Terrill ${ }^{1}$ \\ ${ }^{1}$ Department of Chemistry, San Jose State University, One Washington Square, San Jose, CA 95192-0101, USA \\ ${ }^{2}$ Department of Chemistry and Biochemistry, University of California at Santa Cruz, 1156 High Street, Santa Cruz, CA 95064, USA
}

Correspondence should be addressed to Roger H. Terrill; roger.terrill@sjsu.edu

Received 12 May 2015; Accepted 15 June 2015

Academic Editor: Nicolae Leopold

Copyright (C) 2015 Priya Bhatia et al. This is an open access article distributed under the Creative Commons Attribution License, which permits unrestricted use, distribution, and reproduction in any medium, provided the original work is properly cited.

Gold core/silver shell (Au@Ag) nanoparticles of $\sim 37 \pm 5 \mathrm{~nm}$ diameter generate intense SERS $\left(\lambda_{\mathrm{EX}}=785 \mathrm{~nm}\right)$ responses in solution when they interact with the SERS labels rhodamine 6G (R6G), 4-mercaptopyridine (MPY), and 4-mercaptobenzoic acid (MBA). Herein the relationship between SERS intensity, aggregation, and adsorption phenomenon isobserved by titrating Au@Ag with the above labels. As the labels adsorb to the Au@Ag, they drive aggregation as evidenced by the creation of NIR extinction peaks, and the magnitude of this NIR extinction (measured at $830 \mathrm{~nm}$ ) correlates very closely to magnitude of the intense SERS signals. The label MBA is an exception since it does not trigger aggregation nor does it result in intense SERS; rather intense SERS is recovered only after MBA coated Au@Ag is aggregated with KCl. An "inner filter" model is introduced and applied to compensate for solution extinction when the exciting laser radiation is significantly attenuated. This model permits a summary of the SERS responses in the form of plots of SERS intensity versus the aggregate absorption at $830 \mathrm{~nm}$, which shows the excellent correlation between intense SERS and LSPR bands extinction.

\section{Introduction}

Over the past few decades, the advent of surface enhanced Raman scattering (SERS) has proven enormously powerful for the spectral detection of certain molecules [1]. In a recent example, fruit peels with trace amounts of the pesticide thiram were sprayed with a nanoparticle solution and analyzed in situ yielding surprisingly low detection limits [2]. This simple preparation permitted SERS detection of the unique thiram spectrum directly from the fruit specimens using an affordable semiconductor laser-based $(785 \mathrm{~nm})$ detection system. But the sensitivity of SERS is demonstrated most dramatically by single molecule resonance Raman with nanoparticle-aggregate structures [3-6]. Many applications derive from this uniquely sensitive and selective spectral fingerprinting such as in gas phase chemical analysis [7], cellular analysis [8-10], and tip-enhanced [11] spectroscopy (TERS) which permits the collection of Raman spectra accompanying scanning probe microscopy. Yet exploitation of SERS for chemical analysis remains suboptimal in many practical settings because of the specific set of conditions needed to produce the singularly intense emission which accompanies that small minority of very "hot" SERS emitters [12]. Correlated measurements of particle structure and SERS spectra reveal that intense emission is correlated to nanoparticle pairs or small aggregates, likely possessing $\sim 1 \mathrm{~nm}$ interparticle gaps that host the target molecule [13, 14]. Recent examples of this include the development of dimer or trimer nanostructures that show extraordinary enhancement $[15,16]$.

The enhancement mechanisms underpinning the SERS effect are often classified in one of two ways: either electromagnetic (EM) or chemical (CE) in origin. Amplification of incident optical electric fields by localized surface plasmon resonance (LSPR), which appear in noble metal nanoparticles, and of surface asperities of various geometries is essential to the SERS phenomenon [17]. Chemical enhancement may ultimately be a catch-term for numerous contributions including chemical bonding between the adsorbate molecule 
and the metal [18, 19], electron transfer [20], resonance with electronic states [21], and underpinnings related to the inhomogeneity (e.g., lower symmetry) of local fields relative to isotropic radiation [22].

An additional aspect of SERS is that the electric field enhancement of the Raman effect is expected to scale as $\left|E_{I}\right|^{2}\left|E_{R}\right|^{2}$, when both the incident and reradiating fields enjoy similar plasmon enhancement [23-25]. For this reason, the relatively modest plasmonic optical fields, which at certain locations on a nanoparticle or assembly may yield 100 -fold field increases, can therefore potentially yield prodigious $\left(100^{2}\right)\left(100^{2}\right)=10^{8}$-fold total enhancements when both incident $\left(E_{I}\right)$ and Raman shifted $\left(E_{R}\right)$ frequencies are near enough to resonance with the operative LSPR mode to enjoy large enhancement. Therefore, the incident excitation intensity and the shape and resonance wavelength of the nanoparticle play important roles in defining the electric field enhancements induced by LSPR [26]. In most cases, the enhancements in $E_{I}$ and $E_{R}$ are nearly the same because the LSPR bands are broad compared to the Stokes shifts in normal Raman. In this case, the above enhancement may equate to $\sim E_{I}^{8}$. Of particular importance to this work is the observation that intense SERS is not necessarily associated with a large LSPR intensity which is observable in the far field, that is, that LSPR which corresponds to a detectable signal using conventional optics. For example, Kleinman et al., in a single-particle study, observed optimal SERS enhancements at wavelengths between 785 and $830 \mathrm{~nm}$ for a collection of "hot spot" emitters. These wavelengths were much longer than those associated with the LSPR peaks, indicating that a mechanism other than far-field observable LSPR was responsible for the hot spots observed [14].

Nonetheless, intense SERS signals are generally ascribed to field enhancements at the junctions of nanoparticle dimers [13] and trimers and are often larger than those of the single dipolar particle [27-29]. Nanoparticle aggregates are of interest since they should present many such "hot" nanogaps $[29,30]$. Accordingly, surfaces such as "metal films over nanospheres" [31], nanorings [32], and crescents [33] make good surface SERS substrates. Diffusing nanoparticle dimers and small aggregates also produce intense SERS $[15,34]$. In the examples above however, the LSPR spectra clearly indicate the aggregation state of the SERS emitters.

In this paper we document the relationship between NP aggregation (which is simply driven with the SERS labels themselves [35]) and the SERS emission, observed as a function of label concentration. In essence, this is a SERS titration because the titrant (SERS label) is added gradually while monitoring the SERS signals. Similar "titrimetric" approaches have been explored with an eye to optimizing photothermal ablation therapies [36] tailoring the interaction of amino acids with Au particles [37] and in the context of understanding the SERS response of larger aggregates [38]. In this latter report the temporal aspect of aggregation in a kinetically limited setting (using biotin/avidin) is studied, and the increase and subsequent decrease in SERS intensities are modeled using a variety of methods including electrodynamics and DLVO and DLS calculations and indicate maximum enhancement from small clusters (dimers and trimers). In contrast, this report presents a direct correlation between SERS and aggregate extinction which has not been presented before to our knowledge. However, Kleinman et al. recently showed that a direct correlation between aggregate extinction and SERS is not supported by single-particle studies [14]. In this case, the far-field observable scattering spectra $\left(\lambda_{\text {MAX }} \sim\right.$ $585 \mathrm{~nm}$ ) do not predict $\lambda_{\text {MAX }}$ for the SERS EF, which appears to peak at $785 \mathrm{~nm}$ and then declines gradually. So, in this report, the direct and clear correlation between the aggregate extinction and the SERS signal of the particles may prove controversial.

For the present report, the choice of Au@Ag core-shell nanoparticles is related to the favorable SERS intensities associated with these particles $[2,39]$ as well as their suitability to the $785 \mathrm{~nm}$ excitation source. The titrimetric approach used permits measurements of the correlation between the amounts of titrant used, the intense SERS observed, and the optical extinction spectra (and thereby the aggregation state of the Au@Ag) [40]. Lastly a variety of SERS labels (titrants), rhodamine 6G (R6G), 4-mercaptopyridine (MPY), and 4mercaptobenzoic acid (MBA) (which alone does not trigger aggregation but can later be aggregated via titration with $\mathrm{KCl}$ solution), illustrate general agreement and impart a degree of generality to the conclusions. These conclusions are that the intensity of the SERS response invariably correlates to the aggregate absorption band measured at $830 \mathrm{~nm}$, whether this band appears gradually, abruptly, or following the addition of label and then of the non-SERS active aggregant $\mathrm{KCl}$.

\section{Experimental}

2.1.Preparation of Au@Ag Nanoparticles.Au@Ag nanoparticles were prepared using a chemical metal reduction procedure following Liu et al. [2] but with some minor refinements which we found to improve particle consistency. Gold nanoparticles were synthesized first following the singlephase water based Turkevich method [41]. The protocol used was as follows: first $98.5 \mathrm{mg}$ of hydrogen tetrachloroaurate $\left(\mathrm{HAuCl}_{4}\right.$, Acros, reagent grade) is added to $2.5 \mathrm{~mL}$ of well-purified water $(>10 \mathrm{M} \Omega \mathrm{cm}$, recirculating Millipore polisher/deionizer with $4.5 \mu \mathrm{m}$ filter, used throughout) to prepare a $0.10 \mathrm{M}$ stock solution; a silver nitrate (Acros Ultrapure) stock solution $(1.15 \mathrm{mM})$ is similarly prepared and stored at $4^{\circ} \mathrm{C}$ for no more than 30 days before use. Trisodium citrate (Acros, $98 \%, 3.40 \mathrm{mM}$, and $25 \mathrm{~mL}$ ) and ascorbic acid (Fischer, 99.8\%, 1.00 M, and $25 \mathrm{~mL}$ ) solutions were prepared freshly for each synthesis. A reflux apparatus $(250 \mathrm{~mL}$, threeneck round bottom flask with condenser) cleaned with aqua regia containing a Teflon coated stir bar is then charged with $100 \mathrm{~mL}$ ultrapure water and then $295 \mu \mathrm{L} \mathrm{HAuCl}_{4}$ (added via syringe fitted with a $0.2 \mu \mathrm{m}$ nylon filter tip). Next, $1.56 \mathrm{~mL}$ of trisodium citrate solution is added via filter-tipped syringe to the vigorously stirred, room-temperature $\mathrm{HAuCl}_{4}$ solution. The solution is stirred at room temperature for 15 minutes before heating to $100^{\circ} \mathrm{C}$ which is maintained for $60 \mathrm{~min}$. At that time a color change from a pale yellow to a ruby red is observed. Stirred solutions are allowed to cool to room 
temperature over 30 minutes. At this point, $1 \mathrm{~mL}$ aliquots of the Au core solutions were removed for UV-visible extinction measurement (Cary-50 Bio, 200-1000 nm) and the extinction band shapes and peak wavelengths $\left(\lambda_{\text {MAX }} \sim 518 \mathrm{~nm}\right)$ checked to confirm their conformity to expected values. Ag shell formation was done either immediately following Au core preparation or following overnight storage at $4^{\circ} \mathrm{C}$. Shells are added as follows: first, $7.5 \mathrm{~mL}$ of ascorbic acid solution is added via filter-tipped syringe as above, and, then, $15 \mathrm{~mL}$ of $\mathrm{AgNO}_{3}$ solution is gradually infused via syringe pump and through a nylon filter at a rate of $3.15 \mathrm{~mL}$ per hour, thus triggering a gradual reduction of $\mathrm{Ag}$ onto the $\mathrm{Au}$ cores [2]. All solutions are benchmarked for SERS activity using R6G (standard protocol) at time of preparation and before other experimentation in order to ensure consistency. The above producesAu@Ag nanoparticles of predominantly spherical shape with a diameter of approximately $37 \pm 5 \mathrm{~nm}$ as seen in high resolution TEM images (Figure 2, courtesy of SJSU Professor Folarin Erogbogbo at the UCSC MACS Facility at NASA-AMES).

Stock solutions of rhodamine 6G (R6G, Acros Organics), 4-mercaptopyridine (MPY, Sigma-Aldrich), and 4-mercaptobenzoic acid (MBA, Sigma-Aldrich) at $1,0.1, \ldots$, $0.001 \mathrm{mM}$ were prepared by serial dilution of a $10 \mathrm{mM}$ stock solution yielding solutions labeled A, B, C, D, and E, respectively. All solutions are diluted with highly purified and filtered water as described above.

Raman spectra were collected using an Enwave Optronics EZ-Raman (785 nm, $300 \mathrm{~mW}$ max power) spectrometer in a backscattering geometry and at an optical resolution of $\sim 7 \mathrm{~cm}^{-1}$ and set to approximately $150 \mathrm{~mW}$. Spectra shown are the average of four 4-second scans. The lens tube used has a $7.0 \mathrm{~mm}$ focal length positioned such that the optical path was $6.0 \mathrm{~mm}$ into the liquid volume of the cuvette. Visible absorbance spectra were obtained either concurrently or just following Raman collection using an absorbance spectrometer. The absorption setup employs an Ocean Optics LS1 tungsten halogen light source, coupled via a $400 \mu \mathrm{m}$ multimode fiber optic cable to a pair of collimating lenses and analyzed using an Ocean Optics USB 650 spectrometer. The data acquisition, storage, and analysis were done using a custom software routine (National Instruments LabView 8.2). A schematic of this system is provided in Figure 1.

Spectroscopic titration experiments were conducted by serial additions of small aliquots of SERS label to a wellstirred cuvette using micropipettes. The protocol is as follows: $1.00 \mathrm{~mL}$ of as-prepared nanoparticle solutions is diluted with an equal volume of water into a clean silica cuvette, which is vigorously stirred $(6 \mathrm{~mm} \times 3 \mathrm{~mm}$ Teflon coated magnetic stir bar, ca. 600 RPM) throughout. Absorbance and SERS spectra are recorded at an interval corresponding to $60 \mathrm{~s}$ following the addition of SERS label (e.g., R6G) and using a dedicated set of pipettors (Fisher Pipetman) to improve reproducibility of experiments. Titrations comprised a series of injections of $2.0,3.2,5.0,8.0$, and $12.6 \mu \mathrm{L}$, respectively, for each titrant solution in increasing order of concentration, $1.0,10,100,1000$, and 10,000 $\mu \mathrm{M}$. This produces a log-linear series of increasing quantities of label with only a small

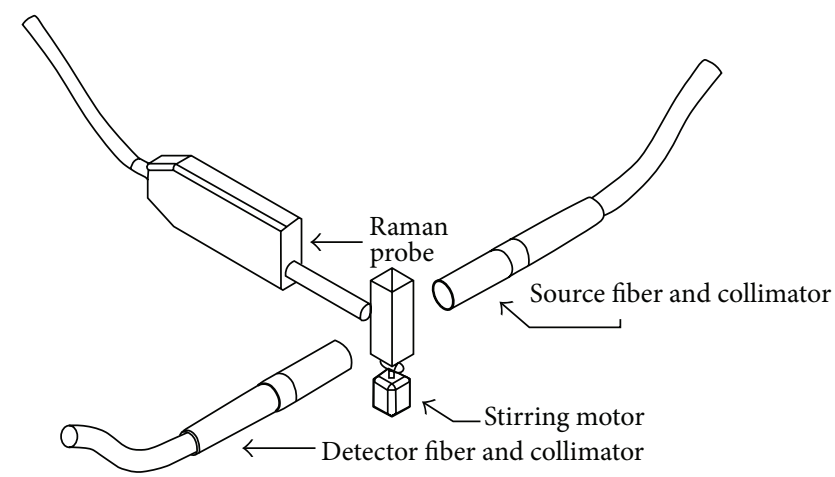

FIGURE 1: Schematic of the experimental apparatus. A fast-stirred fluorescence cuvette is interrogated by both a backscattering Raman probe and a transmission visible extinction probe. The two focal points (Raman and visible) are offset by several $\mathrm{mm}$.

total volume change. Using this protocol it was possible to generate a series of concentrations ranging from $1 \mathrm{nM}$ and up to $250 \mu \mathrm{M}$ in a sequence of 25 steps. After each addition of titrant, a one-minute adsorption time was allowed followed by the acquisition of the Raman and the UV-Vis spectra. The timing of the acquisitions of SERS and UVVis spectra was originally serial (SERS first) and later was done simultaneously. Numerous iterations of all experiments were performed yielding consistent results in terms of general trend, although in some cases the peak SERS signals were much smaller.

\section{Results and Discussion}

Raman spectral results for titration experiments are shown in Figure 3. The spectral positions are consistent with those in literature reports for R6G [25], 4-MPY [42], and 4-MBA [43]. R6G SERS (top) spectra often reached a high intensity over a quite small range of concentrations. Of the 26 spectra shown (covering $0.001 \mu \mathrm{M}$ to $250 \mu \mathrm{M}$, label concentration), detectable SERS is first observed at $1 \mu \mathrm{M}$ (total added label); then the intensity peaks sharply at $2 \mu \mathrm{M}$ and gradually declines as [R6G] concentrations increase. Throughout this range ( 2 to $200 \mu \mathrm{M})$, relative SERS band intensities remain roughly constant. At their peak, these signals correspond to an enhancement factor [44] of

$$
\mathrm{EF} \sim 1.7 \times 10^{6}=\frac{I_{\text {SERS }} / N_{\text {Surf }}}{I_{\text {Raman }} / N_{\text {Vol }}},
$$

where $I_{\text {SERS }}$ and $I_{\text {Raman }}$ are the integrated band intensities and $N_{\text {Surf }}$ and $N_{\text {Vol }}$ are the numbers of molecules interrogated in each setting, respectively. It shows the enhanced SERS signal over the normal Raman signal [44].

The absolute scattering cross section can be estimated by using the absolute cross section of R6G (measured at $\lambda_{\mathrm{EX}}=$ $633 \mathrm{~nm}$ but corrected to $785 \mathrm{~nm})$ as $1.25 \times 10^{-21} \mathrm{~cm}^{2} \mathrm{sr}^{-1}$ which we estimated as below. 


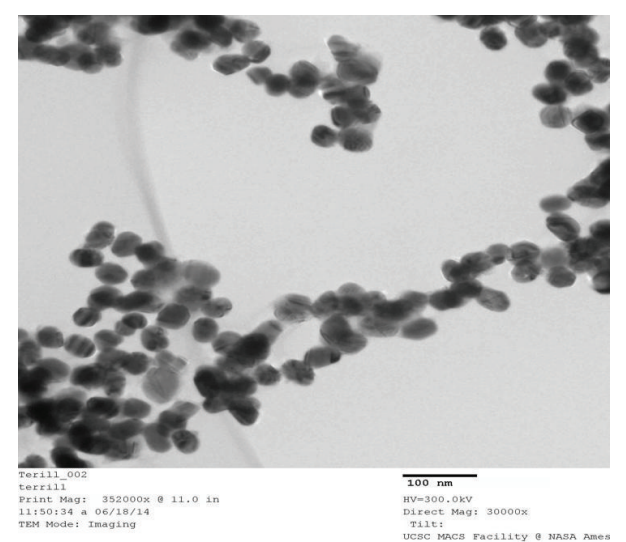

FIGURE 2: Transmission electron microscopy (TEM) images of the Au@Ag nanoparticles.
To estimate the absolute cross sections, we have used a literature value for the $1364 \mathrm{~cm}^{-1}$ band of R6G $(1.8 \times$ $10^{-27} \mathrm{~cm}^{2} \mathrm{sr}^{-1}$, measured using $\lambda_{\mathrm{EX}}=633 \mathrm{~nm}$ ) and the following relation [45]:

$$
\frac{d \sigma_{\text {SERS }} / d \Omega}{d \sigma_{\text {Raman }} / d \Omega}=\frac{I_{\text {SERS }} / C_{\text {SERS }}}{I_{\text {Raman }} / C_{\text {Raman }}},
$$

where $d \sigma_{\text {SERS }} / d \Omega$ and $d \sigma_{\text {Raman }} / d \Omega$ are the differential SERS and normal cross sections of the R6G band in question. $I_{\text {SERS }}$ and $I_{\text {Raman }}$ are the integrated signal intensities for SERS and normal Raman measurements made under identical intensity and integration times. Since in the present case (both diffusing particles and R6G) $C_{\text {SERS }}=N_{\text {Surf }} / V_{\text {Det }}$ and $C_{\text {Raman }}=N_{\text {Vol }} / V_{\text {Det }}\left(V_{\text {Det }}\right.$ is the same in each case because detection geometries are identical) then it follows that

$$
\frac{d \sigma_{\mathrm{SERS}}}{d \Omega}=\mathrm{EF} \times \frac{d \sigma_{\mathrm{Raman}}}{d \Omega} .
$$

The normal Raman differential cross sections for the $1364 \mathrm{~cm}^{-1}$ band of R6G [44] for $\lambda_{\mathrm{EX}}=633 \mathrm{~nm}$ must be corrected for the $\sim \nu^{4}$ frequency dependence of cross section on excitation frequency [44] given by

$$
\sigma \propto \nu_{\mathrm{EX}}\left(\nu_{\mathrm{EX}}-\nu_{\mathrm{Vib}}\right)^{3}
$$

where $\nu_{\mathrm{EX}}$ is the excitation frequency and $\nu_{\mathrm{Vib}}$ is the frequency of the Raman vibrational mode. The correction factor is therefore

$$
\frac{\sigma_{785}}{\sigma_{633}}=\frac{\nu_{\mathrm{EX}}^{785}\left(\nu_{\mathrm{EX}}^{785}-1364 \mathrm{~cm}^{-1}\right)^{3}}{\nu_{\mathrm{EX}}^{633}\left(\nu_{\mathrm{EX}}^{633}-1364 \mathrm{~cm}^{-1}\right)^{3}}=0.40 .
$$

And therefore the absolute cross section of R6G comes out to

$$
\begin{aligned}
\frac{d \sigma_{\text {SERS }}^{785}}{d \Omega} & =0.40 \times \mathrm{EF} \times \frac{d \sigma_{\text {Raman }}^{633}}{d \Omega} \\
& =0.40 \times 1.74 \times 10^{6} \times 1.8 \times 10^{-27} \mathrm{~cm}^{2} \mathrm{sr}^{-1} \\
& =1.2{ }_{5} \times 10^{-21} \mathrm{~cm}^{2} \mathrm{sr}^{-1}
\end{aligned}
$$

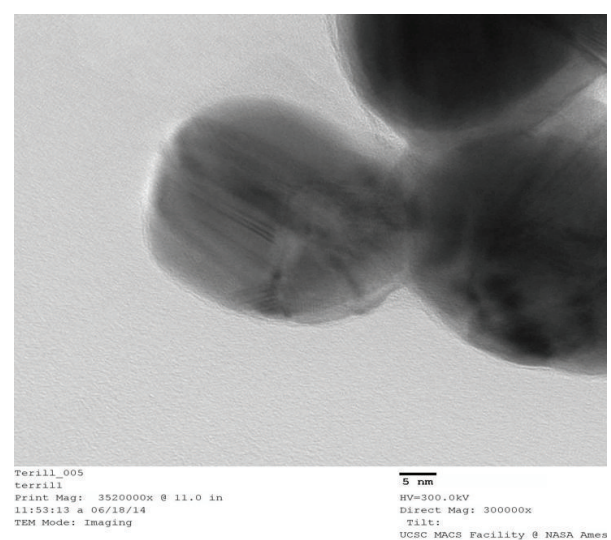

In the case of MPY (Figure 3, middle), the SERS response is both more gradual and more sensitive at low concentrations. For example, the MPY SERS spectrum is clearly detectable at $0.001 \mu \mathrm{M}$ (first injection), then steadily grows until about $0.4 \mu \mathrm{M}$, and then gradually declines. MBA spectra (bottom), in contrast, never become highly intense in this setting but rather begin to generate a weak but detectable signal near $1 \mu \mathrm{M}$ which then gradually increases, reaching a peak intensity just below $10 \mu \mathrm{M}$, but with signal levels $\sim 100$ times lower than that observed for MPY. Selected peak wavenumbers for these three analytes are indicated in Figure 3. Both the overall spectral fingerprints and the peak values agree well with literature reports of SERS using these molecules [13, 46, 47].

Figure 4 illustrates the corresponding optical (VIS-NIR, 400-1000 nm) extinction (absorbance plus scattering) spectra. The spectra are consistent with literature reports of similarly prepared Au@Ag: broad extinction at wavelengths less than $500 \mathrm{~nm}$ and a distinct, nearly Gaussian peak at $\sim 515 \mathrm{~nm}$ attributable to the dipolar localized surface plasmon resonance of noninteracting Au@Ag nanoparticles [2]. Over the course of the titrations, an exchange of extinction intensity was observed as the dipolar LSPR band decreased and a new and progressively broadening band, which originally appeared at $\lambda \sim 750-1000 \mathrm{~nm}$, arose. This band presumably indicates nanoparticle dimers and higher aggregates. These spectral changes closely followed the SERS onsets. As was the case for SERS signals, R6G addition did not noticeably alter the optical spectra until [R6G] reached $\mu \mathrm{M}$ levels, at which point the transformation from monomeric to aggregate spectra began abruptly. Optical spectra of the nanoparticle dipolar band were obscured above [R6G] $\sim 1 \mu \mathrm{M}$ because the strong R6G absorption at $515 \mathrm{~nm}$ overlapped the dipolar LSPR band. Notwithstanding this problem, NIR extinction was observed to evolve, increasing in overall intensity, broadening and red-shifting with each addition of R6G up to the maximum $(2 \mu \mathrm{M})$ concentration observed. MPY optical results were similar but far more gradual in onset. As extinction at 515 decreased steadily, NIR bands arose and red-shifted. Addition of MBA did not lead to gross spectral changes in contrast to both R6G and MPY but MBA did (see below) give rise 

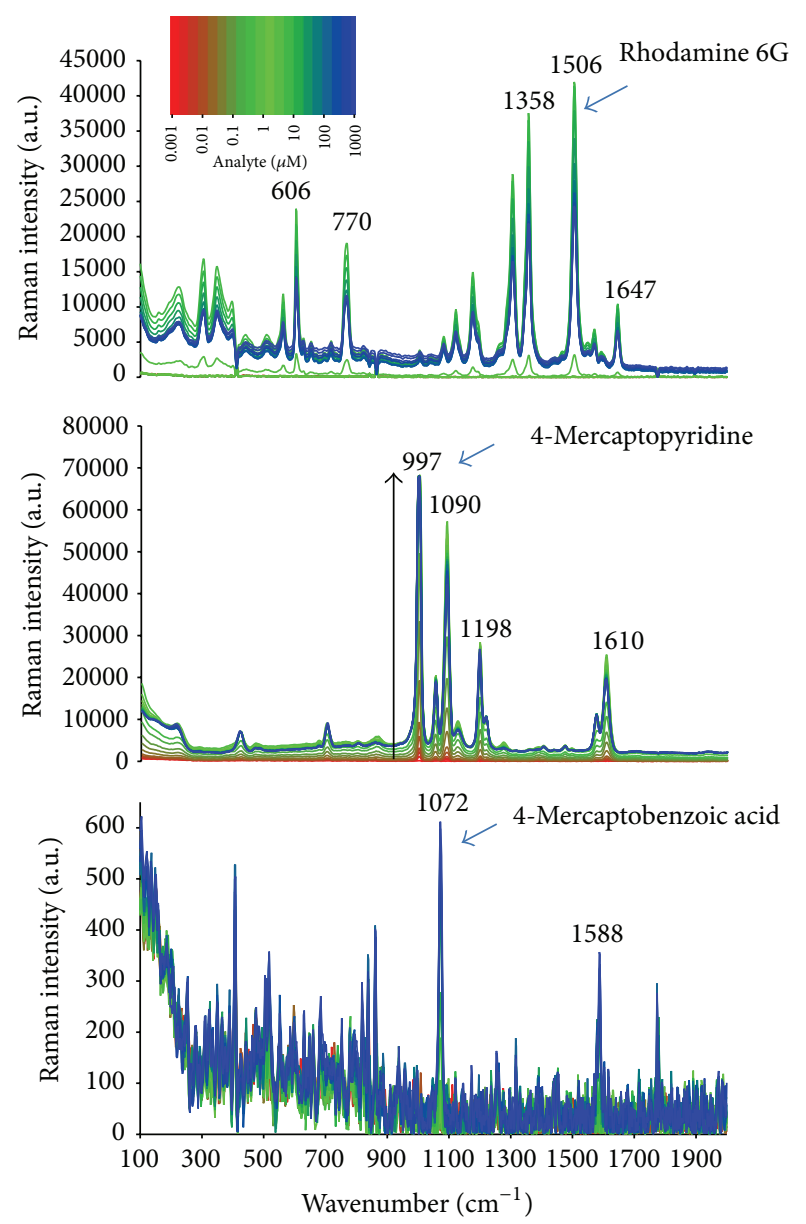

FIGURE 3: Raman scattering spectra of titrants (SERS labels) rhodamine 6G (R6G, top), 4-mercaptopyridine (MPY, middle), and 4mercaptobenzoic acid (MBA, bottom) as these are titrated into ca. 1nMAu@Ag core-shell nanoparticles. Titrant concentrations are as indicated in color bar and range from $1 \mathrm{nM}$ (red) to $1 \mathrm{mM}$ (blue). Peaks are consistent with values reported in the literature [56]. The blue arrows indicate the peaks used for SERS integral intensity calculations.

to subtle shifts in $\lambda_{\mathrm{SPR}}$. Interestingly, there appears to be an approximately isosbestic point near $560 \mathrm{~nm}$ (see circle in Figure 4, middle), indicating a possible direct exchange from monomeric nanoparticles to some new aggregate state or states that have similar extinction at this wavelength.

The important spectral changes: rising SERS intensities (left ordinate) alongside rising NIR extinction (right) are displayed versus label concentration in Figure 5. The two figures clearly change synchronously as titrant is added. The abrupt transition in SERS intensity for R6G is accompanied by an abrupt change in the NIR extinction of nanoparticle aggregates in the solution.

In the case of R6G, the abrupt transition from monomeric nanoparticles to aggregate is hypothesized to be an aggregation event. To calculate the approximate barrier to aggregation for R6G, DLVO theory has been used and it has been employed recently in the nanoparticle context [48]. DLVO theory treats colloid stability in terms of a balance of attractive
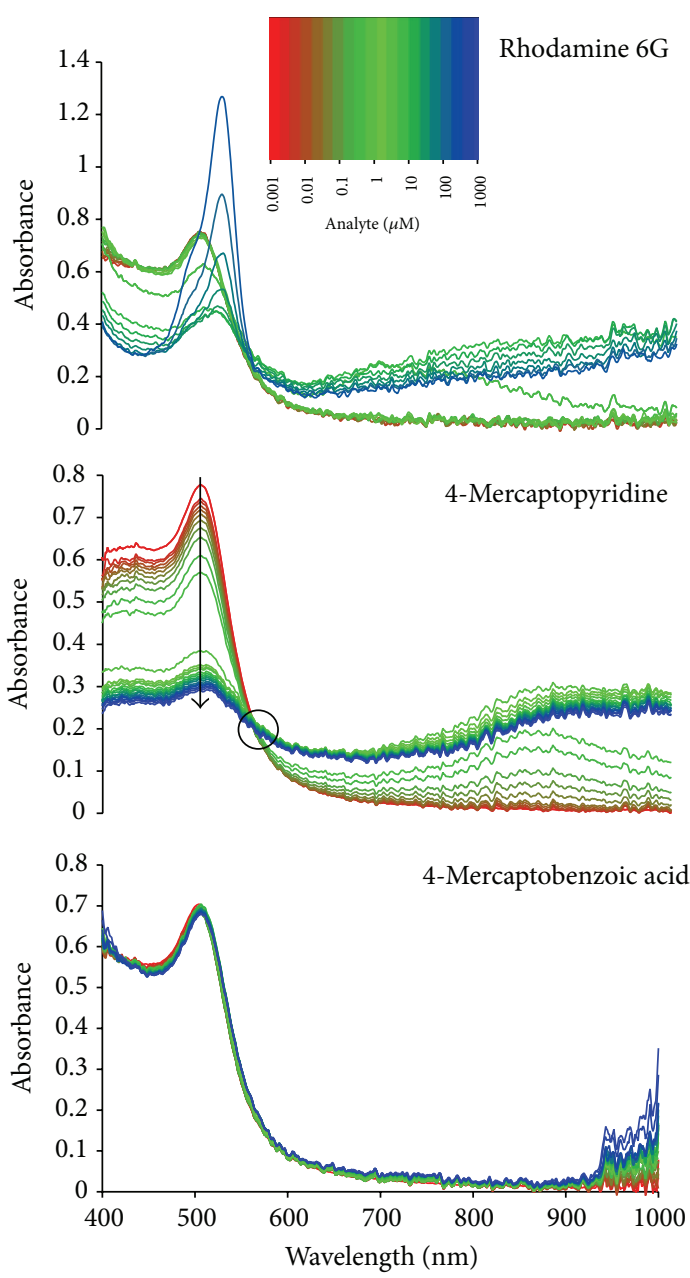

FIGURE 4: Extinction spectra of Au@Ag nanoparticle solutions as a function of added titrants (SERS labels): R6G, top; MPY, middle; and MBA, bottom. Titrant concentrations are color-coded as above and spectra were acquired concurrently with the Raman spectra.

(e.g., van der Waals) forces and repulsive (e.g., electrical double layer) forces. Hence, DLVO provides a framework for understanding the crucial phenomenon of nanoparticle aggregation, a major determinant of SERS phenomena.

The first term in the DLVO barrier calculation is the electrostatic repulsive energy for the two spheres of radius $a$ with zeta-potential $(\zeta)$ approaching each other in a medium of Debye length $\kappa^{-1}$, with $r$ as center to center distance, and is given by

$$
V_{\text {rep }}(k T)=4 \pi \varepsilon_{r} \varepsilon_{0} \zeta \frac{a^{2}}{r} \exp \left[-\kappa a\left(\frac{r}{a}-2\right)\right] .
$$

Above $k$ is the Boltzmann constant, $T$ is the temperature, $\varepsilon_{r}$ is the relative permittivity of water, and $\varepsilon_{0}$ is the vacuum permittivity. The Debye length, $\kappa$, is given by

$$
\kappa^{2}=\frac{2 n^{\infty} e^{2} z^{2}}{\varepsilon_{r} \varepsilon_{0} k T} .
$$

Here $z$ is the valency of the ions comprising the double layer ( \pm 1 in the present case), $e$ is the electronic charge, and $n^{\infty}$ 

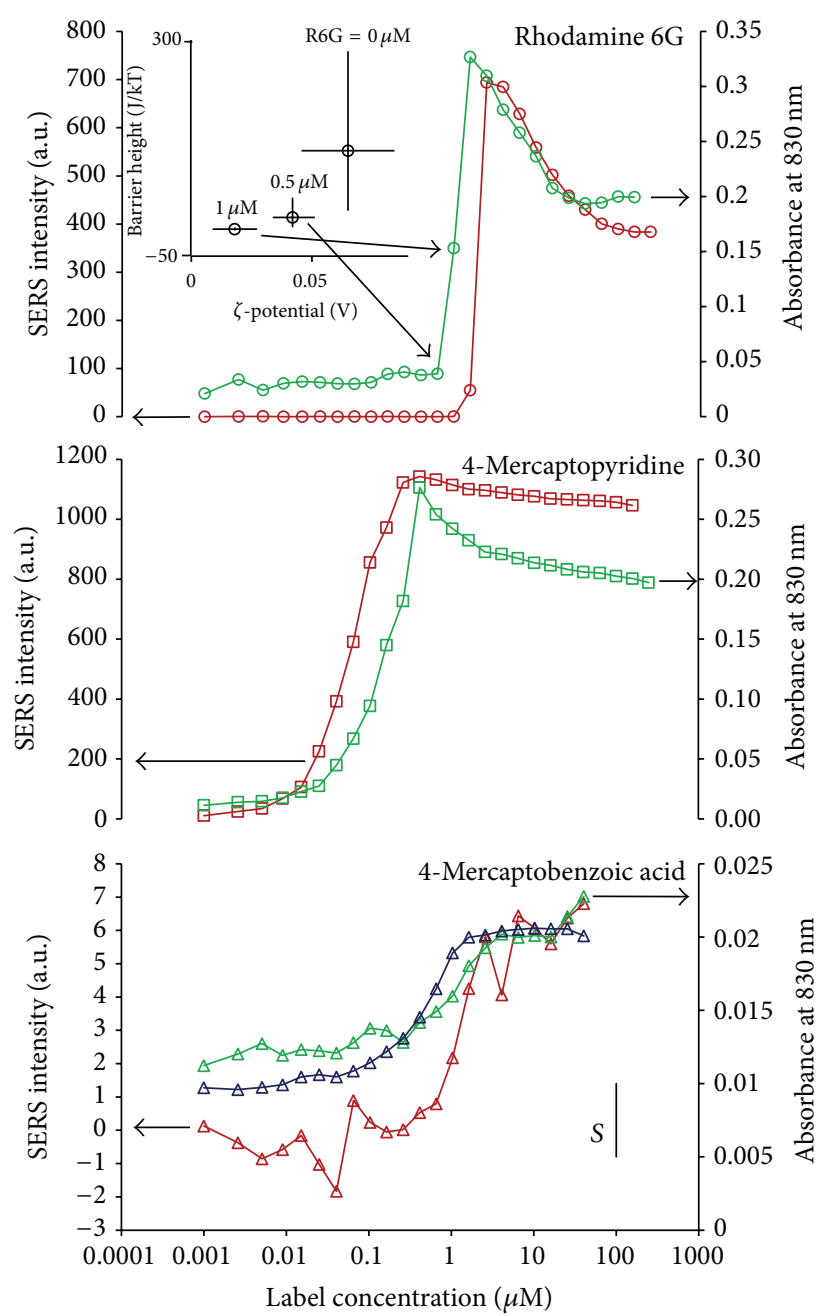

FIGURE 5: Variation of integrated Raman intensity (left scale, red symbols) alongside aggregate-band extinction (right scale, green symbols) as a function of SERS label titrant concentration; R6G (circles, top), MPY (squares, middle), and MBA (triangles, bottom). The bottom panel also includes the wavelength corresponding to variation in $\lambda_{\mathrm{MAX}}$ for the localized surface plasmon (blue, triangles). The scale bar indicates a red-shift (up) of $1 \mathrm{~nm}$. The upper left inset shows the variation of computed DLVO barrier to particle aggregation as a function of the estimated $\zeta$-potential for three nanoparticle specimens incubated with different concentrations of R6G (0.0, 0.5, and $1 \mu \mathrm{M})$.

is the number density of ion in bulk solution which was estimated in this work from solution conductivity using the following relation [49]:

$$
\sigma=\Lambda c z,
$$

where $\sigma$ is the specific conductivity (measured by conductivity probe (Cole Parmer, 19815-00)) of the particle solution, $\Lambda$ is the equivalent conductivity of the electrolyte solute $(\mathrm{NaCl}$ was used in this case), and $c$ is the electrolyte concentration, and, within the bounds of this estimate, also $c=\mu$, the solution ionic strength. In this estimate of $\mu$, all conductivity is attributed to $\mathrm{NaCl}$, which admittedly neglects differences in $\Lambda$ between $\mathrm{NaCl}$ and other less abundant constituents such as hydrogen ion, nitrate, citrate, and ascorbate. However, given the measured $\mathrm{pH}=3.55$ and negligible calculated concentrations of other ions, this estimate is expected to have only a minor impact on the $\zeta$-potential calculation. Therefore, as only minor approximations are necessitated, and considering the constant $\mathrm{pH}$ and conductivity observed in the NP solutions, there is little reason to expect that the trend in the magnitude of the DLVO energy barrier should derogate severely from that predicted here.

In order to estimate changes in the $\zeta$-potential of Au@Ag nanoparticles as they adsorb R6G, experiments were performed to measure their electrophoretic mobility. In these simple experiments, $50 \mu \mathrm{L}$ injections of nanoparticles were pipetted into a quiescent solution of citrate buffer (adjusted to the same $\mathrm{pH}$ and ionic strength as in the optical measurements) in a commercial gel electrophoresis apparatus (EmbiTec Run-One containing only aqueous buffer and no gel). The electrophoretic mobility of the particles was measured under a $64 \mathrm{~V} / \mathrm{cm}$ electric field by observing the drift of the center of the particle aliquots using a video camera. The resulting mobility was then used to calculate the electrophoretic mobility of the particle specimens and, in turn, to calculate the $\zeta$-potential of the particles by applying the Smoluchowski equation, which relates the zeta-potential $(\zeta)$ with the electrophoretic mobility $\left(\mu_{e}\right)$ :

$$
\mu_{e}=\frac{\varepsilon_{r} \varepsilon_{0} \zeta}{\eta}
$$

where $\eta$ is the dynamic viscosity of the dispersion medium, where $\mu_{e}=v / E$, the ratio of drift velocity of the particle to the applied electric field.

The second term in the DLVO expression is the attractive van der Waals interaction energy between particles of radius $a$ and is given by

$$
V_{\text {att }}(k T)=-\frac{A}{6}\left[\frac{2 a^{2}}{r^{2}-4 a^{2}}+\frac{2 a^{2}}{r^{2}}+\ln \left(1-\frac{2 a^{2}}{r^{2}}\right)\right] .
$$

Above $A$ is the Hamaker constant. The interaction between the two particles can be expressed by combining the above described two terms:

$$
V_{\text {tot }}(k T)=V_{\text {rep }}(k T)+V_{\text {att }}(k T) .
$$

Using accepted values of $A$ [48], plots of $V_{\text {tot }}$ versus distance were made for a variety of $\zeta$ values, which correspond to different measured $[\mathrm{R} 6 \mathrm{G}]$. For larger $\zeta$ values, a peak in the energy could be seen as the distance decreases toward contact (Figure 6). This peak is responsible for the stability of the colloid solution, and as $\zeta$ decreases, the peak disappears, and nanoparticle solutions are expected to aggregate. It was found, as expected, that as $\zeta$ decreases, so does the barrier, disappearing near $\zeta=40 \mathrm{mV}$.

Therefore the conclusion that spectral changes are the result of aggregation is supported by DLVO theory as described above. The inset in the upper left corner of Figure 5 illustrates DLVO calculations derived from electrophoretic mobility measurements which allowed us to estimate how 


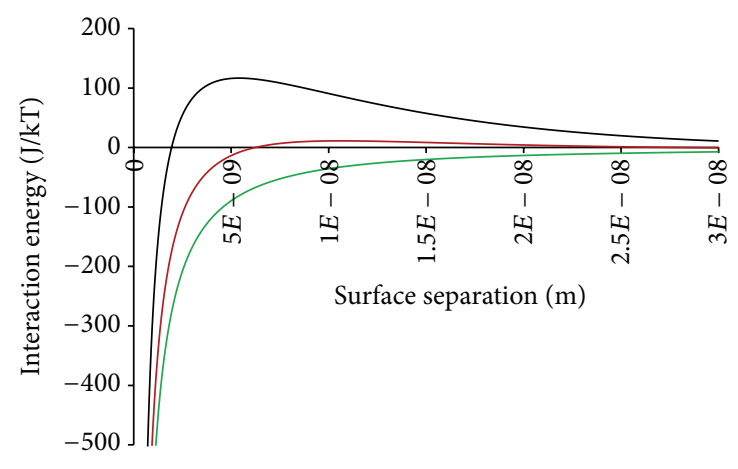

- At $\zeta$-potential $=65 \mathrm{mV},[\mathrm{R} 6 \mathrm{G}]=0.0 \mu \mathrm{M}$
$-42 \mathrm{mV}, 0.5 \mu \mathrm{M}$
$-18 \mathrm{mV}, 1.0 \mu \mathrm{M}$

FIgURE 6: The theoretical potential energy relative to $\mathrm{kT}$ versus surface separation is plotted below for clean nanoparticles and ones preincubated in 0.5 and $1 \mu \mathrm{M}$ R6G. These clearly show the trend towards aggregation and clearly indicate that aggregation is expected for the $1 \mu \mathrm{M}$ case.

the $\zeta$-potential of the particles declined as a function of added R6G in the range corresponding to the abrupt aggregation. For each $[\mathrm{R} 6 \mathrm{G}]$ point in the figure, the approximate barrier to aggregation was computed using DLVO [48]. Therefore, our observations of the spectral signature of aggregation appearing abruptly at $1 \mu \mathrm{M}$ are consistent with the above calculations which predict the collapse of Coulombic barrier at this point.

Similarly, MPY SERS and the nanoparticle NIR LSPR are coincident but rise very gradually and continue to rise over three orders of magnitude in [MPY] (Figure 5, middle). In contrast to the above, MBA SERS signals (bottom) are quite weak. But this is not because MBA fails to adsorb to the Au@Ag. It can be deduced that the MBA molecules are adsorbing by examination of the LSPR wavelength $\left(\lambda_{\mathrm{SPR}}\right)$ as this value is expected to vary as surface adsorption occurs according to the relationship [50]

$$
\Delta \lambda_{\mathrm{SPR}}=m \Delta n\left[1-\exp \left(\frac{-2 d}{l_{d}}\right)\right],
$$

where $m$ is the intrinsic LSPR dielectric sensitivity, $\Delta n$ is the difference in refractive index between the adsorbate and the displaced solvent, $d$ is the adsorbate layer thickness, and $l_{d}$ is the evanescent wave penetration depth of the plasmonic field. As the coverage of adsorbate reaches unity, $\Delta n$ value approaches a limit. In Figure 5, the blue triangular symbols indicate $\Delta \lambda_{\mathrm{SPR}}$ and clearly reveal the expected Langmuirian red-shift expected for the adsorption of a layer of MBA. The absolute magnitude of the shift cannot be fully evaluated without more experimentation with Au@Ag nanoparticles.

In Figure 7 we address the question of why MBA SERS spectra are so weak despite the obvious structural similarity to MPY and given that intense SERS is reported for MBA in the literature in different settings [51]. Our data suggest that, in the setting used, MBA does not trigger aggregation and that the weak SERS signal that we do collect is due to
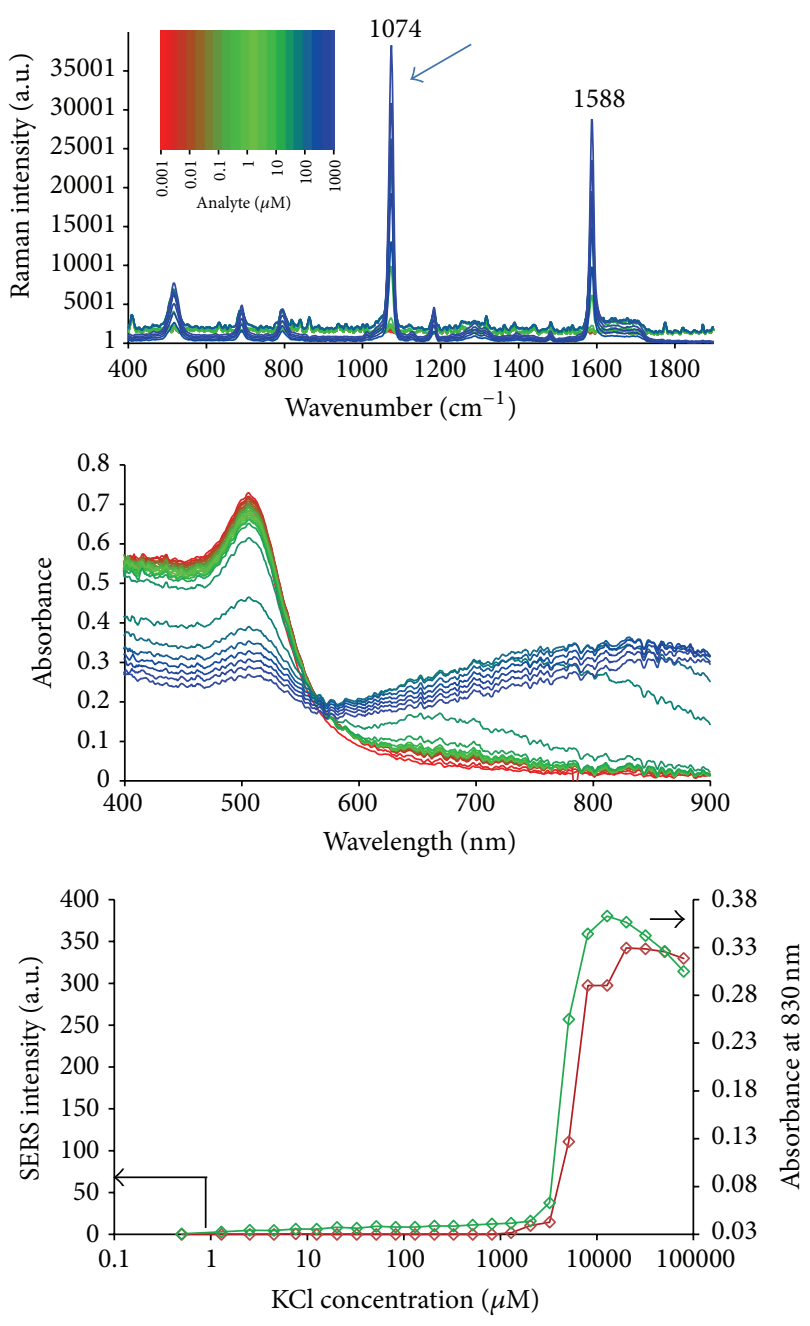

FIGURE 7: In this figure nanoparticle solutions are pretreated with MBA $(1.7 \mu \mathrm{M}$ total concentration) and then titrated with $\mathrm{KCl}$. The top panel is Raman scattering spectra of nanoparticles with preadsorbed MBA. The middle panel shows concurrently acquired extinction spectra. Concentrations are color-coded concentration as above, but now indicating $\mathrm{KCl}$ ranging from $1 \mu \mathrm{M}$ (red) to $100 \mathrm{mM}$ (blue). The figure at the bottom illustrates the variation of integrated Raman intensity (left scale, red diamonds) and aggregate-band extinction (right scale, green diamonds). The blue arrow indicates the band used in SERS intensity calculations.

monomeric Au@Ag coated with MBA. This weak signal, plotted in Figure 5 (bottom), shows a limiting case where the aggregate-associated, highly intense SERS does not appearpresumably a dipolar LSPR underpins these signals. The idea that MBA does not induce aggregation is consistent with the negative free particle $\zeta$-potential for these particles. The cationic dye molecules neutralize the negative potential and trigger aggregation, but the anionic MBA does not. On the other hand, it is possible to aggregate the Au@Ag after coating with MBA. To do this, the particles are pretitrated (in a single $\mathrm{MBA}$ addition) to $[\mathrm{MBA}]=1.7 \mu \mathrm{M}$, corresponding to nearly full surface coverage based on $\Delta \lambda_{\mathrm{SPR}}$ signal, and then a stepwise titration using $\mathrm{KCl}$ as the added titrant is done. $\mathrm{KCl}$ is 
chosen both because of its promotion of SERS signals [18] and because it promotes aggregation by increasing the solution ionic strength. Figure 7 top and middle portions illustrate the SERS intensities and optical extinction spectra as a function of $\mathrm{KCl}$ concentration to the Au@Ag [MBA] system. The onset of SERS signals appears abruptly at $[\mathrm{KCl}] \sim 3 \mathrm{mM}$ and quickly peaks at $[\mathrm{KCl}] \sim 20 \mathrm{mM}$. This very sudden transition is similar to the case of R6G. Optical extinction in the NIR increases in a closely corresponding way, trading intensity from the dipolar plasmon band into aggregate bands over approximately the same range of concentrations. These NP plasmon spectra resemble the MPY progression to a degree, suggestive of an approximately isosbestic point near 560 to $570 \mathrm{~nm}$.

All of the above observations suggest that the intense SERS observed is connected to the aggregation state. Since the relative aggregate concentration can be measured approximately by the absorbance in the NIR region, it makes sense to plot the SERS signals as a function of this absorbance. But the SERS intensity data need to be corrected for light attenuation for both the incoming (785 nm excitation) and outgoing (emitted Stokes Raman wavelengths). These corrections to the SERS output intensity were made to compensate for solution absorption of both the $785 \mathrm{~nm}$ Raman excitation beam and the Stokes shifted emission and are similar to those described for the correction of primary and secondary absorption effects in fluorescence spectroscopy in the analytical chemistry text by Holler et al. [52] but simplified by consideration of emission intensity arising only from the focal point of the excitation laser.

This approximation is justified by the optical configuration of the Raman probe. The collection optics focus the emission from the laser focal point onto the end of a fiber optic, thereby behaving as a spatial filter and therefore rejecting radiation emanating from other points in space within the cuvette. The calculation is facilitated because, in these experiments, we have made concurrent and in situ measurements of the solution absorption at both $\lambda_{\mathrm{EX}}=785 \mathrm{~nm}$ and the Stokes Raman wavelength, $\lambda_{\mathrm{EM}}$, for each SERS measurement. The focal point of the $785 \mathrm{~nm}$ laser excitation beam lies at a point approximately $6 \mathrm{~mm}$ within the solution contained in the cuvette. (This consideration includes the $7 \mathrm{~mm}$ focal length of the lens tube minus $1 \mathrm{~mm}$ for the cuvette wall and a small setback between the focusing lens and cuvette walls.) Therefore we may confidently compute the attenuation of the input laser experienced in the solution at the focal point, which according to Beer's law is

$$
I_{x}^{\mathrm{fp}}=I_{0}^{\mathrm{fp}} 10^{-\epsilon_{\mathrm{EX}} \mathrm{Cx}},
$$

where $\epsilon_{\mathrm{EX}}$ is molar absorptivity at $\lambda_{\mathrm{EX}}=785 \mathrm{~nm}, C$ is absorber concentration and $x$ is pathlength in solution, $I_{x}^{\mathrm{fp}}$ and $I_{0}^{\mathrm{fp}}$ are the intensities at the focal point and prior to entering the solution.

The emitted Raman radiation arising from this focus $\left(I_{x}^{\text {Raman }}\right)$ is transformed into Stokes Raman emission with a certain efficiency proportional to some power, $n$, of the local excitation intensity. This transformation efficiency $\phi$ will include various factors related to the physical states of the nanoparticle-label systems and is what we desire to

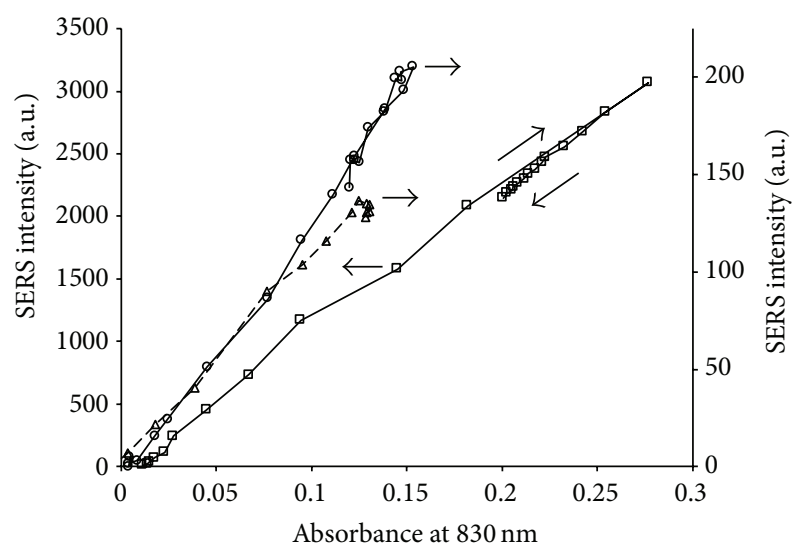

FIGURE 8: In this figure corrected SERS intensities are plotted against the corresponding aggregate extinction data (measured at $830 \mathrm{~nm}$ ) for R6G (circles), MPY (squares), and MBA titrated with $\mathrm{KCl}$ (triangles). R6G and MBA are associated with the right $y$-axis and MPY is associated with the left.

characterize as a function of added label in the titration experiments. The intensity of the emitting species in the beam focus will be

$$
I_{x}^{\text {Raman }}=\phi\left(I_{x}^{\mathrm{fp}}\right)^{n} .
$$

But, we must consider the attenuation of this Raman emission by the solution as it travels from the focus to the collection lens on the Raman probe. This distance is the same as that transited by the excitation beam and the emitted beam is attenuated according to the absorptivity $\epsilon_{\mathrm{EM}}$ at the Stokes (EM) wavelength yielding a measurable intensity $\left(I_{0}^{\text {Raman }}\right)$ :

$$
I_{0}^{\text {Raman }}=I_{x}^{\text {Raman }} 10^{-\epsilon_{\mathrm{EM}} C x} .
$$

The measured attenuated Raman intensity can be related to the emission process at the focus by combining the above:

$$
I_{0}^{\text {Raman }}=\phi\left(I_{0}^{\mathrm{fp}} 10^{-\epsilon_{\mathrm{EX}} \mathrm{C} x}\right)^{n} 10^{-\epsilon_{\mathrm{EM}} \mathrm{C} x} .
$$

Since our aim in this study is to recover this intrinsic activity term $\phi\left(I_{0}^{\mathrm{fp}}\right)^{n}$ as the solution is titrated and becomes more opaque, we can correct the measured signals by dividing by the attenuation factors to produce an absorption corrected value $\left(I_{\text {Corr }}^{\text {Raman }}\right)$ :

$$
I_{\text {Corr }}^{\text {Raman }}=\phi\left(I_{0}^{\mathrm{fp}}\right)^{n}=\frac{I_{0}^{\text {Raman }}}{\left(10^{-\epsilon_{\mathrm{EX}} C x}\right)^{n} 10^{-\epsilon_{\mathrm{EM}} C x}} .
$$

The exponents $\epsilon_{\mathrm{EX}} C x$ and $\epsilon_{\mathrm{EM}} C x$ are derived directly from the concurrently acquired extinction measurements. The power dependence of SERS emission, $n$, is assumed to be 2, consistent with current $|E|^{4}$ models of plasmon field enhancement of the incident and emitted beams [23].

Figure 8 illustrates representative corrected $I_{\text {SERS }}$ versus $A_{\text {AGGREGATE }}(830 \mathrm{~nm})$ measurements for the three analytes. Note that in acquiring these data it is important that the SERS and absorption data are acquired at the same time because of 
the dynamic nature of the aggregation process. The spectral correction is also crucial as, without it, SERS is lower at high absorbance and exhibits negative deviation from linearity above $A>0.05-0.1$. This figure summarizes one of the more strikingly consistent aspects of Au@Ag SERS: the intense SERS signals in this system are clearly in direct proportion to the aggregate absorption bands intensities near $830 \mathrm{~nm}$.

\section{Conclusions}

These results underscore the crucial connection between intense SERS and the LSPR signature of aggregation in this colloidal system. This is not surprising, since LSPR connections in surface bound [13] and in the solution phase $[40,53-55]$ have long been known. However, recent singleparticle work has called into serious question the need for farfield observable LSPR [14]. In all of the cases observed (aggregation with labels or with $\mathrm{KCl}$ ), the dipolar LSPR band near $510 \mathrm{~nm}$ exchanges intensity with a broad NIR 750-1000 nm band which corresponds to the longitudinal and coupled plasmon resonances of the nanoparticle dimers and aggregates, respectively. What we have established in this paper is that, for the Au@Ag system, aggregation, as measured by the absorbance at $\sim 830 \mathrm{~nm}$, is consistently connected to the onset and progression of intense SERS. The large enhancement factors that we observed $\left(\sim 10^{6}\right)$ are consistent with the intense SERS being a result of the formation of junction hot spots [30] or higher-order aggregates [13]. The presence of a nearly 100 -fold increase in the SERS signals between nonaggregated and aggregated (with $\mathrm{KCl}$ ) particles in the case of the nonaggregating label MBA further supports the aggregate "rule"; in this case relatively weak SERS correlates with LSPR inferred by MBA surface coverage, but intense SERS is only observed following $\mathrm{KCl}$-induced aggregation.

\section{Conflict of Interests}

The authors declare that there is no conflict of interests regarding the publication of this paper.

\section{References}

[1] J. Z. Zhang, D. A. Wheeler, A. M. Schwartzberg, and J. Shi, "Basics and practice of surface enhanced Raman scattering (SERS) and tip enhanced Raman scattering (TERS)," Biomedical Spectroscopy and Imaging, vol. 3, pp. 121-159, 2014.

[2] B. Liu, G. Han, Z. Zhang et al., "Shell thickness-dependent raman enhancement for rapid identification and detection of pesticide residues at fruit peels," Analytical Chemistry, vol. 84, no. 1, pp. 255-261, 2012.

[3] X.-M. Qian and S. M. Nie, "Single-molecule and single-nanoparticle SERS: from fundamental mechanisms to biomedical applications," Chemical Society Reviews, vol. 37, no. 5, pp. 912920, 2008.

[4] K. Kneipp, Y. Wang, H. Kneipp et al., "Single molecule detection using surface-enhanced Raman scattering (SERS)," Physical Review Letters, vol. 78, no. 9, pp. 1667-1670, 1997.

[5] L. Brus, "Noble metal nanocrystals: plasmon electron transfer photochemistry and single-molecule raman spectroscopy,"
Accounts of Chemical Research, vol. 41, no. 12, pp. 1742-1749, 2008.

[6] J. P. Camden, J. A. Dieringer, Y. Wang et al., "Probing the structure of single-molecule surface-enhanced Raman scattering hot spots," Journal of the American Chemical Society, vol. 130, no. 38, pp. 12616-12617, 2008.

[7] N. Leopold, M. Haberkorn, T. Laurell et al., "On-line monitoring of airborne chemistry in levitated nanodroplets: in situ synthesis and application of SERS-active Ag-sols for trace analysis by FT-raman spectroscopy," Analytical Chemistry, vol. 75, no. 9, pp. 2166-2171, 2003.

[8] E. A. Vitol, Z. Orynbayeva, G. Friedman, and Y. Gogotsi, "Nanoprobes for intracellular and single cell surface-enhanced Raman spectroscopy (SERS)," Journal of Raman Spectroscopy, vol. 43, no. 7, pp. 817-827, 2012.

[9] E. A. Vitol, Z. Orynbayeva, M. J. Bouchard, J. Azizkhan-Clifford, G. Friedman, and Y. Gogotsi, "In situ intracellular spectroscopy with surface enhanced raman spectroscopy (SERS)-enabled nanopipettes," ACS Nano, vol. 3, no. 11, pp. 3529-3536, 2009.

[10] W. Xie, L. Su, A. Shen, A. Materny, and J. Hu, "Application of surface-enhanced Raman scattering in cell analysis," Journal of Raman Spectroscopy, vol. 42, no. 6, pp. 1248-1254, 2011.

[11] B. Pettinger, B. Ren, G. Picardi, R. Schuster, and G. Ertl, "Nanoscale probing of adsorbed species by tip-enhanced Raman spectroscopy," Physical Review Letters, vol. 92, no. 9, Article ID 096101, 2004.

[12] Y. Fang, N.-H. Seong, and D. D. Dlott, "Measurement of the distribution of site enhancements in surface-enhanced raman scattering," Science, vol. 321, no. 5887, pp. 388-392, 2008.

[13] K. L. Wustholz, A.-I. Henry, J. M. McMahon et al., "Structureactivity relationships in gold nanoparticle dimers and trimers for surface-enhanced raman spectroscopy," Journal of the American Chemical Society, vol. 132, no. 31, pp. 10903-10910, 2010.

[14] S. L. Kleinman, B. Sharma, M. G. Blaber et al., "Structure enhancement factor relationships in single gold nanoantennas by surface-enhanced raman excitation spectroscopy," Journal of the American Chemical Society, vol. 135, no. 1, pp. 301-308, 2013.

[15] G. B. Braun, S. J. Lee, T. Laurence et al., "Generalized approach to SERS-active nanomaterials via controlled nanoparticle linking, polymer encapsulation, and small-molecule infusion," The Journal of Physical Chemistry C, vol. 113, no. 31, pp. 13622-13629, 2009.

[16] A.-I. Henry, J. M. Bingham, E. Ringe, L. D. Marks, G. C. Schatz, and R. P. Van Duyne, "Correlated structure and optical property studies of plasmonic nanoparticles," Journal of Physical Chemistry C, vol. 115, no. 19, pp. 9291-9305, 2011.

[17] I. Freitag, U. Neugebauer, A. Csaki, W. Fritzsche, C. Krafft, and J. Popp, "Preparation and characterization of multicore SERS labels by controlled aggregation of gold nanoparticles," Vibrational Spectroscopy, vol. 60, pp. 79-84, 2012.

[18] D. Pristinski, S. Tan, M. Erol, H. Du, and S. Sukhishvili, "In situ SERS study of Rhodamine 6G adsorbed on individually immobilized Ag nanoparticles," Journal of Raman Spectroscopy, vol. 37, no. 7, pp. 762-770, 2006.

[19] S. K. Ghosh and T. Pal, "Interparticle coupling effect on the surface plasmon resonance of gold nanoparticles: from theory to applications," Chemical Reviews, vol. 107, no. 11, pp. 47974862, 2007.

[20] M. Moskovits, "Surface-enhanced spectroscopy," Reviews of Modern Physics, vol. 57, no. 3, article 783, 1985. 
[21] A. Otto, "The 'chemical' (electronic) contribution to surfaceenhanced Raman scattering," Journal of Raman Spectroscopy, vol. 36, no. 6-7, pp. 497-509, 2005.

[22] M. Moskovits, "Surface-enhanced Raman spectroscopy: a brief retrospective," Journal of Raman Spectroscopy, vol. 36, no. 6-7, pp. 485-496, 2005.

[23] P. L. Stiles, J. A. Dieringer, N. C. Shah, and R. P. Van Duyne, "Surface-enhanced raman spectroscopy," Annual Review of Analytical Chemistry, vol. 1, no. 1, pp. 601-626, 2008.

[24] A. Campion and P. Kambhampati, "Surface-enhanced Raman scattering," Chemical Society Reviews, vol. 27, no. 4, pp. 241-250, 1998.

[25] A. M. Michaels, M. Nirmal, and L. E. Brus, "Surface enhanced Raman spectroscopy of individual rhodamine 6G molecules on large Ag nanocrystals," Journal of the American Chemical Society, vol. 121, no. 43, pp. 9932-9939, 1999.

[26] J. Z. Zhang and C. Noguez, "Plasmonic optical properties and applications of metal nanostructures," Plasmonics, vol. 3, no. 4, pp. 127-150, 2008.

[27] K. L. Kelly, E. Coronado, L. L. Zhao, and G. C. Schatz, "The optical properties of metal nanoparticles: the influence of size, shape, and dielectric environment," The Journal of Physical Chemistry B, vol. 107, no. 3, pp. 668-677, 2003.

[28] K. Li, M. I. Stockman, and D. J. Bergman, "Self-similar chain of metal nanospheres as an efficient nanolens," Physical Review Letters, vol. 91, no. 22, Article ID 227402, 2003.

[29] A. M. Michaels, J. Jiang, and L. Brus, "Ag nanocrystal junctions as the site for surface-enhanced Raman scattering of single Rhodamine 6G molecules," Journal of Physical Chemistry B, vol. 104, no. 50, pp. 11965-11971, 2000.

[30] A. M. Schwartzberg, C. D. Grant, A. Wolcott et al., "Unique gold nanoparticle aggregates as a highly active surface-enhanced raman scattering substrate," The Journal of Physical Chemistry $B$, vol. 108, no. 50, pp. 19191-19197, 2004.

[31] P. Freunscht, R. P. van Duyne, and S. Schneider, "Surfaceenhanced Raman spectroscopy of trans-stilbene adsorbed on platinum- or self-assembled monolayer-modified silver film over nanosphere surfaces," Chemical Physics Letters, vol. 281, no. 4-6, pp. 372-378, 1997.

[32] J. Aizpurua, P. Hanarp, D. S. Sutherland, M. Käll, G. W. Bryant, and F. J. García de Abajo, "Optical properties of gold nanorings," Physical Review Letters, vol. 90, no. 5, Article ID 057401, 2003.

[33] R. Bukasov, T. A. Ali, P. Nordlander, and J. S. ShumakerParry, "Probing the plasmonic near-field of gold nanocrescent antennas," ACS Nano, vol. 4, no. 11, pp. 6639-6650, 2010.

[34] T. A. Laurence, G. Braun, C. Talley et al., "Rapid, solution-based characterization of optimized SERS nanoparticle substrates," Journal of the American Chemical Society, vol. 131, no. 1, pp. 162$169,2009$.

[35] H. Bengter, C. Tengroth, and S. P. Jacobsson, "New light on Agcolloid preparation for surface-enhanced FT-raman spectroscopy: the role of aggregation," Journal of Raman Spectroscopy, vol. 36, no. 11, pp. 1015-1022, 2005.

[36] H. Kitching, A. J. Kenyon, and I. P. Parkin, "The interaction of gold and silver nanoparticles with a range of anionic and cationic dyes," Physical Chemistry Chemical Physics, vol. 16, no. 13, pp. 6050-6059, 2014.

[37] G. H. Mihailescu, L. Olenic, S. Pruneanu, I. Bratu, and I. Kacso, "The effect of $\mathrm{pH}$ on amino acids binding to gold nanoparticles," Journal of Optoelectronics and Advanced Materials, vol. 9, no. 3, pp. 756-759, 2007.
[38] J. C. Fraire, L. A. Pérez, and E. A. Coronado, "Cluster size effects in the surface-enhanced raman scattering response of ag and Au nanoparticle aggregates: experimental and theoretical insight," The Journal of Physical Chemistry C, vol. 117, no. 44, pp. 23090 23107, 2013.

[39] C. Hoff, Correlation of surface enhanced raman spectroscopy and nanoparticle aggregation with rhodamine $6 G$ [M.S. thesis], San Jose State University, San Jose, Calif, USA, 2013.

[40] V. Amendola, O. M. Bakr, and F. Stellacci, "A study of the surface plasmon resonance of silver nanoparticles by the discrete dipole approximation method: effect of shape, size, structure, and assembly," Plasmonics, vol. 5, no. 1, pp. 85-97, 2010.

[41] J. Kimling, M. Maier, B. Okenve, V. Kotaidis, H. Ballot, and A. Plech, "Turkevich method for gold nanoparticle synthesis revisited," The Journal of Physical Chemistry B, vol. 110, no. 32, pp. 15700-15707, 2006.

[42] J. R. Lombardi and R. L. Birke, "A unified approach to surfaceenhanced raman spectroscopy," The Journal of Physical Chemistry C, vol. 112, no. 14, pp. 5605-5617, 2008.

[43] C. J. Orendorff, A. Gole, T. K. Sau, and C. J. Murphy, "Surfaceenhanced Raman spectroscopy of self-assembled monolayers: sandwich architecture and nanoparticle shape dependence," Analytical Chemistry, vol. 77, no. 10, pp. 3261-3266, 2005.

[44] E. C. Le Ru, E. Blackie, M. Meyer, and P. G. Etchegoint, "Surface enhanced raman scattering enhancement factors: a comprehensive study," The Journal of Physical Chemistry C, vol. 111, no. 37, pp. 13794-13803, 2007.

[45] J. M. Dudik, C. R. Johnson, and S. A. Asher, "Dependence of the preresonance Raman cross sections of $\mathrm{CH}_{3} \mathrm{CN}, \mathrm{SO}_{4}^{2-}, \mathrm{ClO}_{4}^{-}$, and $\mathrm{NO}_{3}^{-}$," The Journal of Chemical Physics, vol. 82, pp. 1732-1740, 1985.

[46] Y. Wang, H. Hu, S. Jing et al., "Enhanced raman scattering as a probe for 4-mercaptopyridine surface-modified copper oxide nanocrystals," Analytical Sciences, vol. 23, no. 7, pp. 787-791, 2007.

[47] L. Xia, Y. Jia, G. Liu et al., "Adjustment and control of SERS activity of metal substrates by pressure," Journal of Raman Spectroscopy, vol. 41, no. 4, pp. 398-405, 2010.

[48] P. Mulvaney, "Metal nanoparticles: double layers, optical properties, and electrochemistry," in Nanoscale Materials in Chemistry, pp. 121-167, John Wiley \& Sons, 2001.

[49] J. O. Bockris and A. K. Reddy, Modern Electrochemistry: An Introduction to an Interdisciplinary Area, vol. 2, Springer, 1973.

[50] K. A. Willets and R. P. van Duyne, "Localized surface plasmon resonance spectroscopy and sensing," Annual Review of Physical Chemistry, vol. 58, pp. 267-297, 2007.

[51] A. Michota and J. Bukowska, "Surface-enhanced raman scattering (SERS) of 4-mercaptobenzoic acid on silver and gold substrates," Journal of Raman Spectroscopy, vol. 34, no. 1, pp. 21$25,2003$.

[52] F. J. Holler, D. A. Skoog, and S. R. Crouch, Principles of Instrumental Analysis, Cole Publishing, Pacific Grove, Calif, USA, 2007.

[53] A. M. Gabudean, D. Biro, and S. Astilean, "Localized surface plasmon resonance (LSPR) and surface-enhanced Raman scattering (SERS) studies of 4-aminothiophenol adsorption on gold nanorods," Journal of Molecular Structure, vol. 993, no. 1-3, pp. 420-424, 2011.

[54] M. Potara, A.-M. Gabudean, and S. Astilean, "Solution-phase, dual LSPR-SERS plasmonic sensors of high sensitivity and 
stability based on chitosan-coated anisotropic silver nanoparticles," Journal of Materials Chemistry, vol. 21, no. 11, pp. 36253633, 2011.

[55] G. C. Schatz, M. A. Young, and R. P. Van Duyne, "Electromagnetic mechanism of SERS," in Surface-Enhanced Raman Scattering, vol. 103 of Topics in Applied Physics, pp. 19-45, Springer, Berlin, Germany, 2006.

[56] A. Sabur, M. Havel, and Y. Gogotsi, "SERS intensity optimization by controlling the size and shape of faceted gold nanoparticles," Journal of Raman Spectroscopy, vol. 39, no. 1, pp. 6167, 2008. 

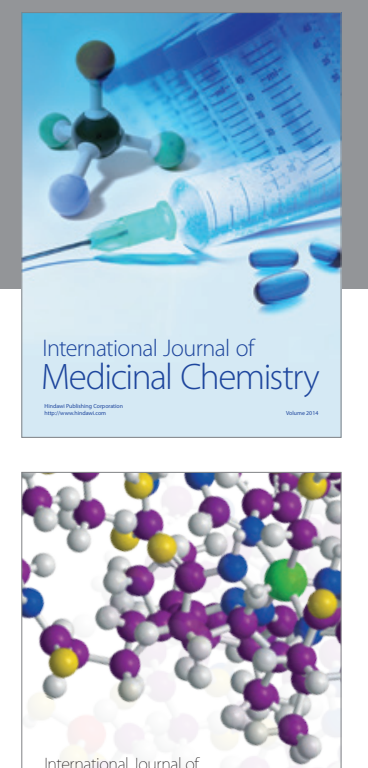

\section{Carbohydrate} Chemistry

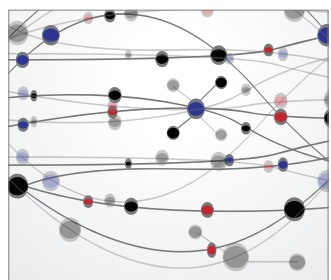

The Scientific World Journal
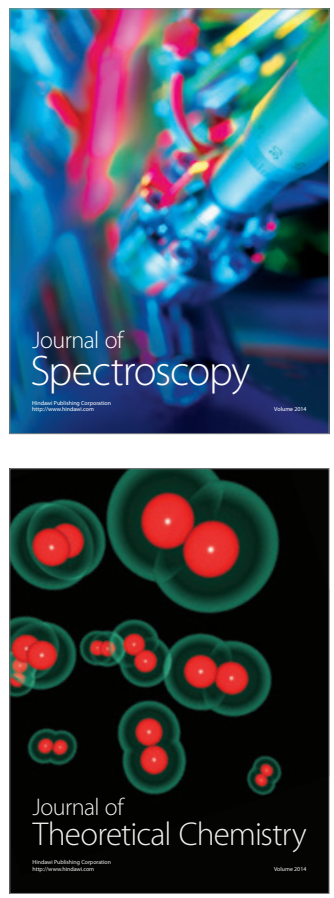
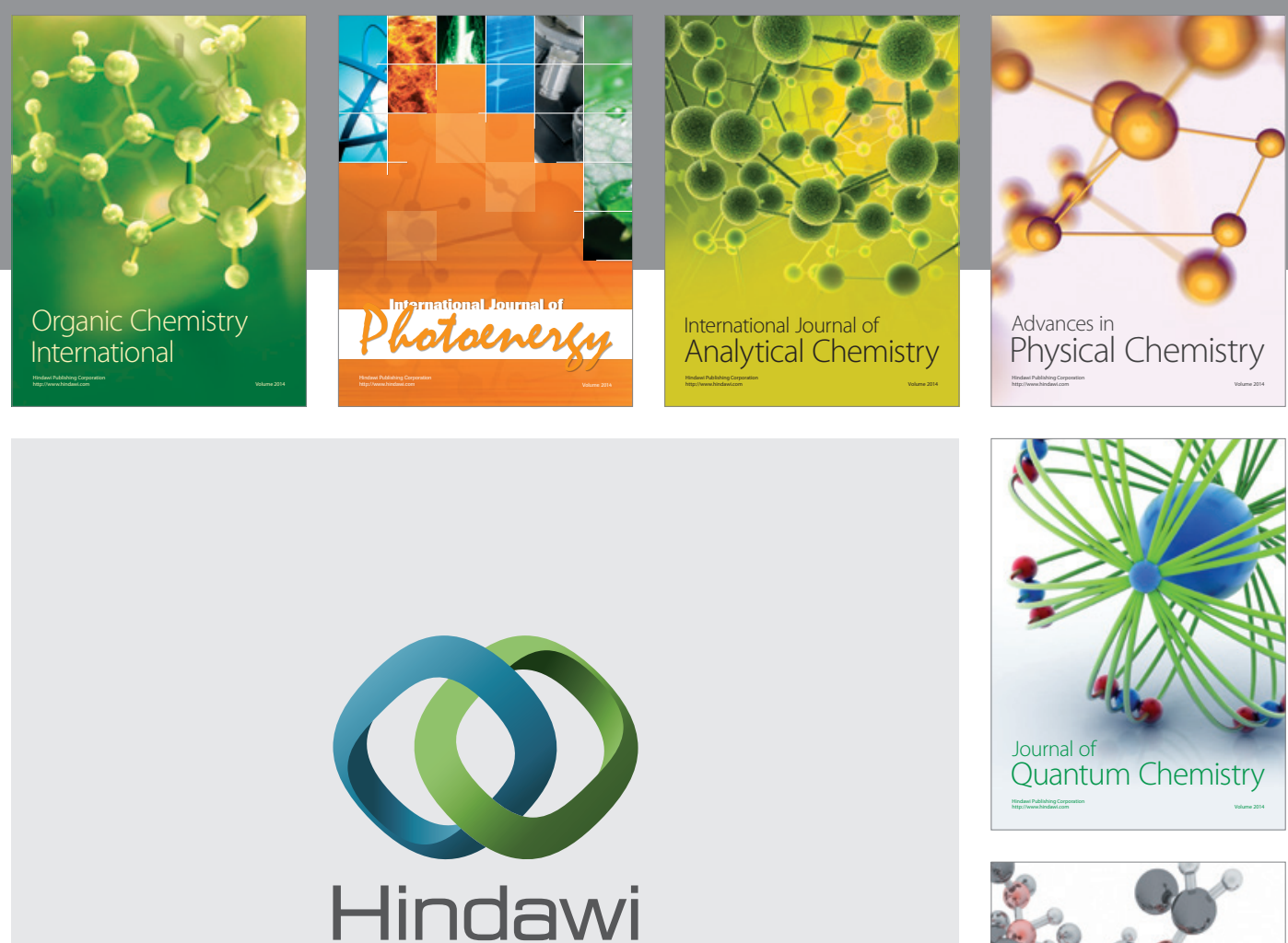

Submit your manuscripts at

http://www.hindawi.com

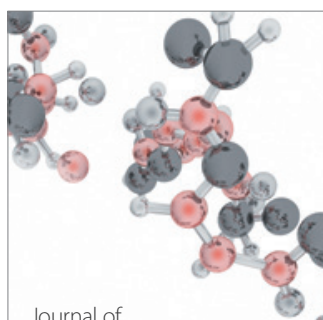

Analytical Methods

in Chemistry

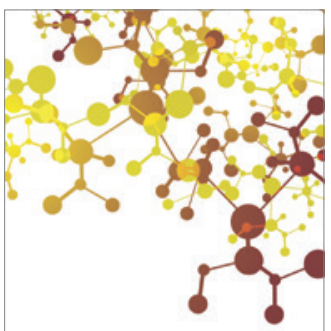

Journal of

Applied Chemistry

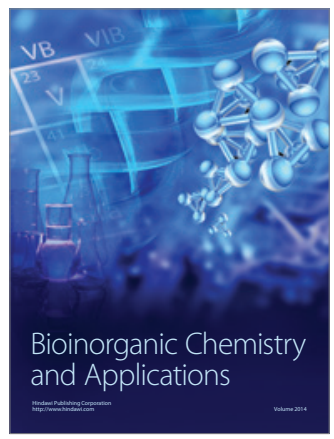

Inorganic Chemistry
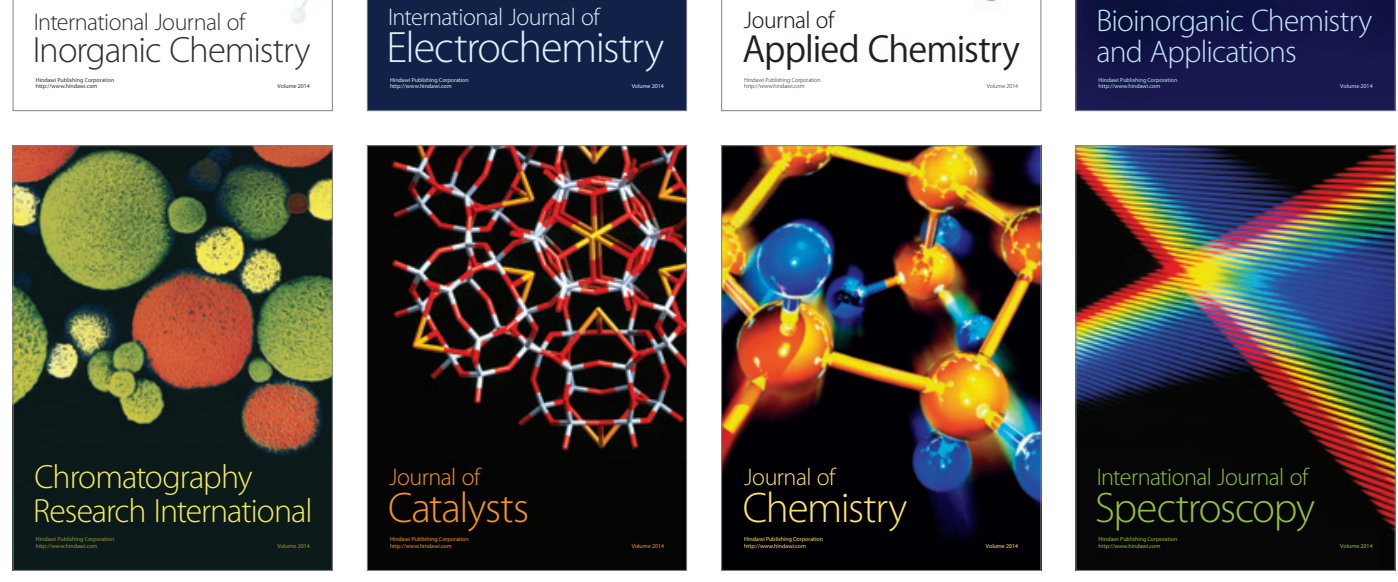